\title{
IMPROVED Ar(II) TRANSITION PROBABILITIES
}

\author{
K. Danzmann ${ }^{\dagger}$ and M. Kock \\ Institut für Plasmaphysik, Universität Hannover, Callinstrasse 38, 300 Hannover 1, West Germany
}

(Received 30 August 1982)

\begin{abstract}
Precise Ar(II) branching ratios have been measured on a high current hollow cathode with a 1-m Fourier transform spectrometer. Absolute transition probabilities for $11 \mathrm{Ar}(\mathrm{II})$ lines were calculated from these branching ratios and lifetime measurements published by Mohamed et al. For the prominent $4806 \AA$ line, the present result is $A_{i k}=7.12 \times 10^{7} \mathrm{~s}^{-1} \pm 2.8 \%$, which is in excellent agreement with recent literature data derived from pure argon diagnostics, two-wavelength-interferometry, and $\mathrm{H}_{\beta}$-diagnostics when using the theoretical data of Vidal et al.
\end{abstract}

\section{INTRODUCTION}

Many values for argon transition probabilities have been presented in the literature during the last two decades. However, discrepancies remain on the order of $30 \%$ between the absolute scales obtained by different methods.

In a recent paper, Baessler and Kock ${ }^{1}$ reported on precise electron-density measurements in a high current cascaded arc by using two-wavelength-interferometry. Under the assumption of local thermodynamic equilibrium (LTE), they determined transition probabilities for the Ar(I) line $4300 \AA$ and the $\operatorname{Ar}($ II) line $4806 \AA$. Their results favor the values derived from pure argon diagnostics as opposed to those which have been derived from $\mathrm{H}_{\beta}$-diagnostics (see, e.g., Preston, ${ }^{2}$ Wiese et al. ${ }^{3}$ ). This fact has been confirmed in Ref. 1 using experimentally determined reduced half and quarter widths of the hydrogen line $\mathrm{H}_{\beta}$, which turned out to be systematically lower than those derived from theoretical treatments. The latter results have been corroborated by Helbig and Nick, ${ }^{4}$ who performed a comparable experiment.

All of these experiments require assumptions on the plasma state when absolute transition probabilities are to be measured. Additionally, in these dense plasmas, the Stark-broadened lines have far reaching wings which have to be included in the intensity measurement (line wing correction).

A method, with which these problems can be avoided, is the combination of lifetime and branching-ratio measurements. During the past decade, the reliability and accuracy of lifetime measurements have been improved considerably by avoiding or excluding cascade effects, so that data having an accuracy of a few percent can be obtained. In order to derive absolute transition probabilities from a lifetime measurement, the complete branching ratio has to be known. Thus, measurements are required on a large spectral range from the u.v. to, e.g., the far i.r.

In the present paper, we report on measurements performed at the Kitt Peak National Observatory, Tucson, Arizona. A high current hollow cathode was used as a very stable light source to record argon spectra over a wide range with the 1-m Fourier spectrometer. ${ }^{5}$ Subsequently, absolute $\operatorname{Ar}(\mathrm{II})$ line transition probabilities are evaluated from these branching ratios and published lifetime data.

\section{RESULTS}

We have recorded five spectra with the 1-m Fourier transform spectrometer. The light source used was a hollow cathode. ${ }^{6}$ Experimental details such as the calibration of the spectra are described elsewhere. ${ }^{25}$

For an absolute calibration, we used the accurate Ar(II) lifetimes published by Mohamed $e t$ $a l^{7}$ The data were obtained by a special delayed coincidence technique which yields data free from cascade effects. The lifetime $\tau_{i}$ of an excited level $i$ is related to the transition probabilities $A_{i k}$ of all lines arising from that level by

$$
\tau_{i}=1 / \sum_{k} A_{i k}
$$

†Present address: Stanford University, Department of Physics, Stanford, CA 94305, U.S.A. 
For the levels ${ }^{4} P_{5 / 2}^{0} \quad(\tau=7.78 \pm 0.12 \mathrm{~ns})$ and ${ }^{4} P_{3 / 2}^{0}(\tau=7.02 \pm 0.03 \mathrm{~ns})$, we computed the wavelengths of all allowed transitions and searched our spectra for them. For all i.r. lines with wavelengths outside the range of our spectra, we calculated branching ratios from the semiempirical $f$-values of Kurucz and Peytreman. ${ }^{8}$ These lines were included in the sum of all branches. In neither case did their contribution exceed $0.7 \%$, since nearly all these transitions are intercombination lines.

The absolute transition probabilities of $11 \mathrm{Ar}(\mathrm{II})$ lines are listed in Table 1. The errors quoted are composed of three contributions. (i) The errors of lifetime measurements ${ }^{7}$ are given above. (ii) The statistical uncertainty $\Delta R_{k} / R_{k}$ of the individual branching ratio $R_{k}=I_{k} / I_{0}$, where $I$ is the relative line intensity, is given by the standard deviation evaluated from five independent runs. (iii) The possible systematic error of the sum $S=\sum_{k} R_{k}$ of all branches is calculated from

$$
\frac{\Delta S}{S}=\left[\sum_{k}\left(\frac{\Delta R_{k}}{R_{k}} \times \frac{R_{k}}{S}\right)^{2}\right]^{1 / 2}
$$

For the branching ratios calculated from the data of Kurucz and Peytreman, we assumed a possible uncertainty of $\pm 100 \%$, giving a contribution to $\Delta S / S$ of $\pm 0.5 \%$.

\section{DISCUSSION}

A comparison with other authors is carried out for the prominent line $4806 \AA$, which has been the subject of many investigations. A graphical representation of this comparison is shown in Fig. 1. The values can be divided into three categories: (i) pure argon diagnostics, best fit, two-wavelength-interferometry; (ii) $\mathrm{H}_{\beta}$-diagnostics by comparing Stark-broadened hydrogen lines with theoretical profiles; (iii) lifetime measurements in connection with measured branch-

Table 1. Ar(II) transition probabilities.

\begin{tabular}{|c|c|c|c|c|}
\hline Upper level & Lower level & $\begin{array}{l}\text { Wave- } \\
\text { length, } \&\end{array}$ & A, $10^{6} \mathrm{~s}^{-1}$ & Error, \\
\hline $4 p^{4} P_{5 / 2}^{0}$ & $\begin{array}{l}3 d{ }^{4} \mathrm{D}_{7 / 2} \\
3 \mathrm{~d}{ }^{4} \mathrm{D}_{5 / 2} \\
3 \mathrm{~d}^{4} \mathrm{D}_{3 / 2} \\
4 \mathrm{~s}{ }^{4} \mathrm{P}_{5 / 2} \\
4 \mathrm{~s}{ }^{4} \mathrm{P}_{3 / 8}\end{array}$ & $\begin{array}{l}4400.99 \\
4431.00 \\
4460.56 \\
4806.02 \\
5009.33\end{array}$ & $\begin{array}{l}30.5 \\
10.7 \\
1.46 \\
71.2 \\
13.8\end{array}$ & $\begin{array}{l}4.4 \\
5.1 \\
3.2 \\
2.8 \\
5.1\end{array}$ \\
\hline $4 \mathrm{p}^{4} \mathrm{P}_{3 / 2}^{0}$ & $\begin{array}{l}3 d{ }^{4} D_{5 / 2} \\
3 d{ }^{4} D_{3 / 2} \\
3 d{ }^{4} D_{1 / 2} \\
4 s^{4} P_{5 / 2} \\
4 s{ }^{4} P_{3 / 2} \\
4 s^{4} P_{1 / 2}\end{array}$ & $\begin{array}{l}4371.33 \\
4400.10 \\
4420.91 \\
4735.91 \\
4933.21 \\
5062.04\end{array}$ & $\begin{array}{l}23.5 \\
17.3 \\
3.24 \\
59.7 \\
14.9 \\
22.9\end{array}$ & $\begin{array}{l}3.3 \\
3.1 \\
4.7 \\
1.9 \\
3.5 \\
2.6\end{array}$ \\
\hline
\end{tabular}




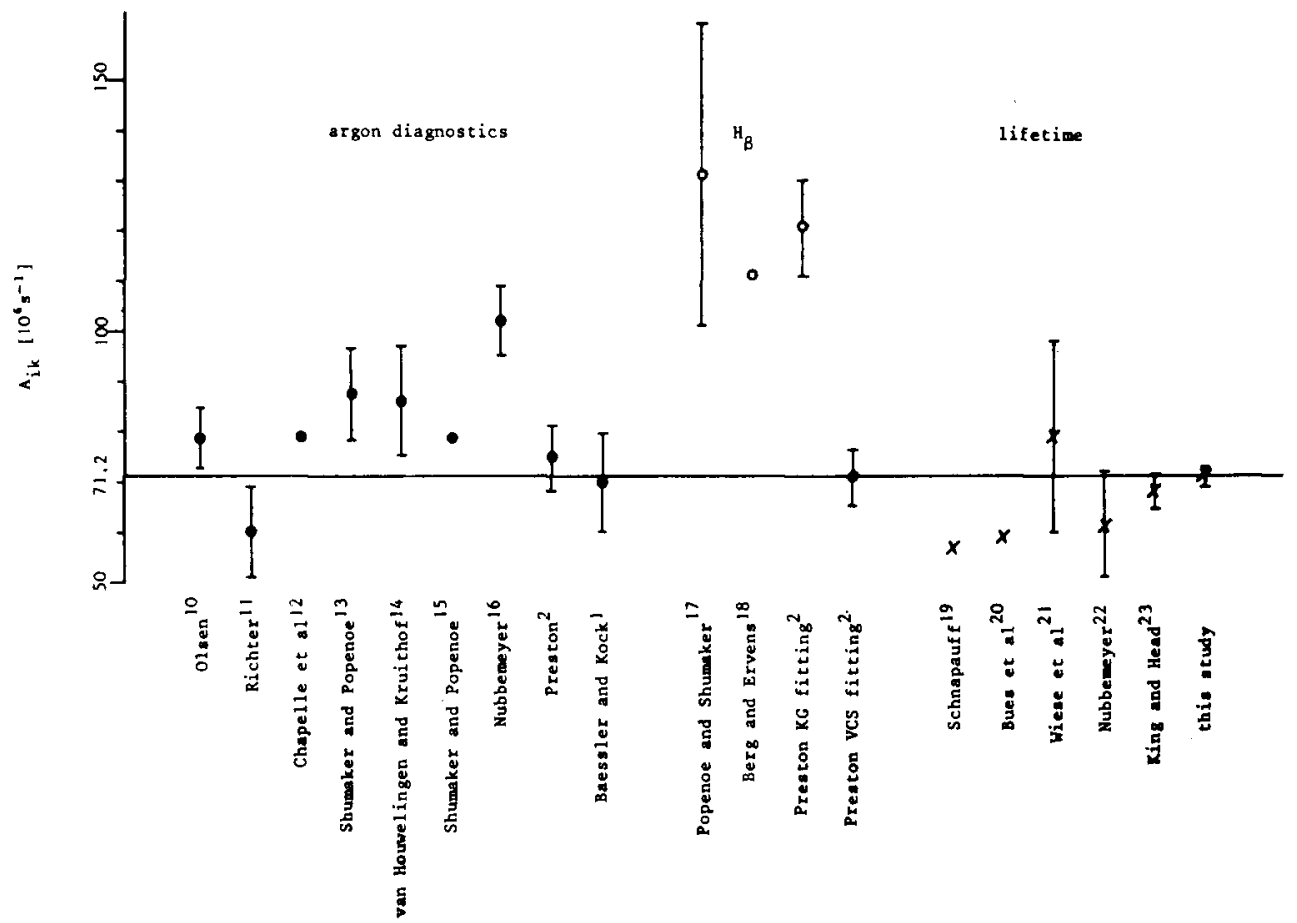

Fig. 1. Transition probability of the Ar(II) line $4806 \AA$ measured by various authors.

ing ratios. Excluding the values based on $\mathrm{H}_{\beta}$-diagnostics, the mean value of all data is $A_{i k}=7.18 \times 10^{7} \mathrm{~s}^{-1}$, which is very close to our value of $A_{i k}=7.12 \times 10^{7} \mathrm{~s}^{-1}$.

We have excluded two values 1.c. from the averaging procedure. The value given by Nubbemeyer ${ }^{16}$ seems to be too large compared to those of other authors who have also applied the best-fit method or laser interferometry. The value tabulated by Wiese et al. ${ }^{21}$ is not the result of an independent measurement but of a critical compilation of the older data shown in Fig. 1.

Preston $^{2}$ gives three different values for this line, depending on the method used to determine the plasma state. For his $\mathrm{H}_{\beta}$-based electron density determination, he applied the theories of Kepple and Griem ${ }^{24}$ and Vidal et al. ${ }^{9}$ (henceforth referred to as KG and VCS, respectively).

The value Preston obtained with the KG theory is too high by about $70 \%$. The $\mathrm{H}_{\beta}$-based data from other authors show similar discrepancies ( $c f$ Fig. 1). However, using the VCS results, Preston obtained a value which is in close agreement with our value. His result obtained from pure argon diagnostics is higher by only $5 \%$ and his error bars include our value.

Baessler and Kock' determined the plasma state by an interferometric electron density measurement. Their result is only $1.7 \%$ lower than ours.

The value in Fig. 1 which is assigned to King and $\mathrm{Head}^{23}$ has been computed by Preston ${ }^{2}$ by renormalizing the transition probabilities of Shumaker and Popenoe ${ }^{13}$ to the lifetime measurements of King and Head. The good agreement with our value indicates that the deviations found for the original data of Shumaker and Popenoe are only due to their determination of the plasma state.

It should be mentioned that line-wing corrections frequently discussed in the literature (e.g. Preston ${ }^{2}$ ) for $\operatorname{Ar}(\mathrm{I})$ lines are less critical for the $\operatorname{Ar}(\mathrm{II}) 4806 \AA$ line. For the present experiments, line wing corrections are negligible since the narrow lines emitted by our hollow cathode plasma are almost purely Doppler-broadened.

\section{CONCLUSION}

The combination of lifetime and branching ratio measurements has yielded accurate transition probabilities without any assumptions concerning the plasma state. 
The advanced techniques of lifetime determination provide data free from systematic errors such as cascade effects. For the measurement of the total set of branching ratios, including all lines from the u.v. to the i.r., a Fourier transform spectrometer connected with a very stable light source offers outstanding opportunities. The determination of the present $\operatorname{Ar}(\mathrm{II})$ transition probabilities with uncertainties less than $5 \%$ illustrate the superiority of this combined method.

Acknowledgements-We would like to thank Kitt Peak National Observatory, Tucson, Arizona, for providing their facilities and Deutsche Forschungsgemeinschaft for financial support. We are indebted to J. M. Brault for continuing interest and generous cooperation. We also thank E. Schulz-Gulde for fruitful discussions.

\section{REFERENCES}

1. P. Baessler and M. Kock, J. Phys. B 13, 1351 (1980).

2. R. C. Preston, J. Phys. B 10, 1377 (1977).

3. W. L. Wiese, M. W. Smith, and D. R. Paquette, Phys, Rev. A 6. 1132 (1972)

4. V. Helbig and K. P. Nick, J. Phys. B 14, 3573 (1981).

5. J. Brault, "Solar Fourier Transform Spectroscopy", Proc. JOSO Workshop, Osservazioni e Memorie dell. Osserv. Astrof. di Arcetri (Firenze) Fascicolo 106, 33 (1978).

6. K. Danzmann and M. Kock, J. Phys. B 13, 2051 (1980).

7. K. A. Mohamed, G. C. King, and F. H. Read, J. Phys. B 9, 3159 (1976).

8. R. L. Kurucz and E. Peytreman, Smiths Astrophys. Obs. Spec. Rep. 362, Cambridge, Mass. (1975)

9. C. R. Vidal, J. Cooper, and E. W. Smith, Astrophys. J. Suppl. No. 214, 25, 37 (1973).

10. H. N. Olsen, JQSRT 3, 59, 305 (1963).

11. J. Richter, Z. Astrophys. 61, 57 (1965).

12. J. Chapelle. A. Sy, F. Cabannes, and J. Blandi, JOSRT 8, 1201 (1968).

13. J. B. Shumaker and C. H. Popenoe, JOSA 59, 980 (1969).

14. D. van Houwellingen and A. Kruithof, JQSRT 11, 1235 (1971).

15. J. B. Shumaker and C. H. Popenoe, J. Res. NBS A 76, 71 (1972).

16. H. Nubbemeyer, JQSRT 16, 395 (1976).

17. C. H. Popenoe and J. B. Shumaker, J. Res. NBS A 69, 495 (1965).

18. H. F. Berg and W. Ervens, Z. Phys. 206, 184 (1967).

19. R. Schnapauff, Z. Astrophys. 68, 431 (1968).

20. J. Bues, T. Haag, and J. Richter, Laboratoriumsbericht Finst. f. Exp. Phys., Universitaet Kiel (1966).

21. W. L. Wiese, M. W. Smith. and B. M. Miles, Nat. Stand. Ref. Data Ser. NBSII (22). 187 (1969).

22. H. Nubbemeyer, Ph.D Thesis Freie Universität Berlin (1974).

23. D. B. King and C. E. Head, Phys. Rev. 13, 1778 (1976).

24. P. Kepple and H. R. Griem, Phys, Rev. 173, 317 (1968).

25. K. Danzmann and M. Kock. JOSA 72, 1556 (1982). 